\title{
Chylaskos as a presentation of serous papillary adenocarcinoma of the endometrium: a case report
}

\author{
Maria Inês Sequeira' ${ }^{1}$ Tiago Borges², Isabel Camões² \\ ${ }^{1}$ Medical Oncology Department, Instituto Português de Oncologia do Porto (Porto, Portugal) \\ 2 Internal Medicine Department, Centro Hospitalar de São João (Porto, Portugal)
}

\begin{abstract}
A 77-year-old female was presented to the emergency department with intense anorexia, weight loss despite progressive abdominal distension, and dyspnea. Abdomen imaging studies revealed moderate-volume ascites and a hepatic space-occupying lesion. Diagnostic paracentesis allowed the drainage of a chylous effusion and cytology analysis identified adenocarcinoma cells. Hepatic metastasis of papillary serous adenocarcinoma of the endometrium was confirmed after tomography-guided biopsy.

Endometrial carcinoma is the most common malignant gynecological neoplasm in developed countries and is often classified in type I with endometrioid histology (estrogen-dependent) and non endometrioid type II (non-estrogen-dependent). Chylous ascites or chylaskos is a rare presentation on hospital admission. Several etiologies have been described. In adults, solid malignancy is expected to be identified in less than $20 \%$ of the cases. Currently only one case of endometrial carcinoma presenting with chylous ascites was described in a systematic review.
\end{abstract}

Keywords: Chylous ascites, endometrial neoplasms

\section{Clinical case}

A 77-year-old female was admitted in the Internal Medicine ward for investigation after presenting to the emergency department with intense anorexia, weight loss despite progressive abdominal distension and dyspnea. As past medical history, the patient related chronic gastritis, chronic microcytic anemia, arterial hypertension, dyslipidemia and type 2 diabetes mellitus with no vascular events, besides chronic kidney disease (stage 3). She denied any alcohol or smoke habits. On gynecological reports she had one term labor with eutocic delivery, menarche at 12 and menopause at 40 years, with no further complaints or bleeding discharge since then. Laboratory evaluation showed microcytic anemia (hemoglobin $7.4 \mathrm{~g} / \mathrm{dL}$, mean corpuscular volume $75.7 \mathrm{fL}$ ) with normal white cells and platelet count, mild hypoalbuminemia (31.6 g/L), normal serum total protein $(67.7 \mathrm{~g} / \mathrm{L})$, normal serum transaminases ( aspartate aminotranferease $17 \mathrm{U} / \mathrm{L}$, alanine aminotranferease $8 \mathrm{U} / \mathrm{L}$ ) normal gamma glutamyl transpeptidase (30 U/L), alkaline phosphatase (110 U/I) and lactate dehydrogenase (228 U/L) values; high erythrocyte sedimentation rate $(71 \mathrm{~mm}$ in the first hour) and a high C-reactive protein (64.3 mg/L), not changed baseline renal function (serum creatinine of $1.60 \mathrm{mg} / \mathrm{dL}$ with an estimated glomerular filtration rate of $35 \mathrm{~mL} / \mathrm{min} / 1.73 \mathrm{~m} 2)$.

Diagnostic paracentesis allowed the drainage of an exudative effusion. The laboratory analysis revealed 574 total cells with lymphocytes predominance (total protein of $51.9 \mathrm{~g} / \mathrm{L}$, albumin of $25.8 \mathrm{~g} / \mathrm{L}$, with a serum-to-ascites albumin gradient (SAAG) of $0.58 \mathrm{~g} / \mathrm{dL}$, lactate dehydrogenase level of $175 \mathrm{IU} / \mathrm{L}$, glucose level of $126 \mathrm{mg} / \mathrm{dL}$ and triglyceride level of $382 \mathrm{mg} / \mathrm{dL}$. It wasn't detected any microorganism from the effusion. These chemistry results were consistent with a chylous effusion. Cytological analysis identified adenocarcinoma cells of unknown origin. Abdominopelvic magnetic resonance imaging revealed several hypodense lesions in both hepatic lobes, suggestive of metastatic disease and moderate-volume ascites and a small bilateral pleural effusion, without adenopathy or another abnormal structures including adnexal glands, spleen or pancreas (Figure 1).
The patient was evaluated by Gynecology and at the physical examination she had a friable endometrium. She underwent transvaginal ultrasound and hysteroscopy with biopsy that showed serous papillary cells with immunoreactivity for p53 protein (100\%), estrogen receptors (ER - 80\%) and progesterone receptors (PR10\%).

Since the presentation of endometrial carcinoma as chylous ascites is a rare entity (see below), we considered the presence of a second neoplasm. After excluding digestive neoplasia with colonoscopy and upper gastrointestinal endoscopy, we performed a tomography-guided biopsy of the hepatic metastasis which corroborated the diagnosis of endometrial adenocarcinoma with a tubulo-papillary pattern, extensive areas of necrosis and positivity for ER, PR, p53 and cytokeratin-7. Finally, endometrial carcinoma was staged according to FIGO (Fédération Internationale de Gynécologie et d'Obstétrique) clas-

Figure 1. Magnetic resonance imaging showing moderate-volume ascites

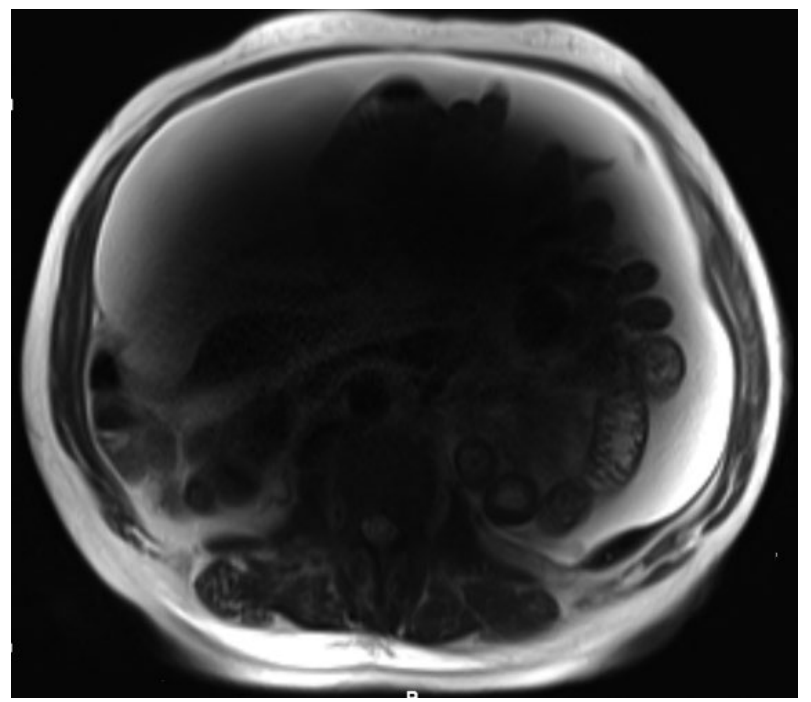


sification (stage IVb based on diffuse nodular hepatic metastases and peritoneal carcinomatosis). The patient was proposed for palliative systemic chemotherapy that she could not receive due to the rapid clinical deterioration because of an obstructive renal failure. The patient died a few weeks after discharged.

\section{Discussion}

Endometrial carcinoma is the fourth most common malignant gynecological neoplasm in developed countries; it is often classified, according to clinic-pathological and molecular characteristics, in type I and II. Type I includes tumor in grade 1 or 2 with endometrioid histology (estrogen-dependent); Type II includes advanced grade 3 endometrioid carcinomas and non endometrioid (non-estrogen-dependent) with serous papillary, clear cell, mucinous, squamous, transitional cells, mesonephric and undifferentiated histology. ${ }^{1,2}$ Type I endometrial carcinoma is associated with exogenous (hormonal therapeutic) or endogenous estrogen (obese and diabetic patient) exposure. This case corresponds to a papillary serous carcinoma (type II, non-estrogen dependent), despite the endogenous estrogen exposure as risk factor. The papillary serous carcinoma represents about $5 \%$ of all endometrial carcinomas. ${ }^{3}$ They have a complex papillary architecture, always have marked nuclear atypia, and are highly aggressive. The diagnosis requires histopathological confirmation, while surgical staging depends on acquisition of peritoneal fluid or washings, exploration of the abdominal cavity and pelvic and para-aortic nodal areas, and total hysterectomy with bilateral salpingo-oophorectomy. ${ }^{2}$ About $75 \%$ of endometrial tumors are limited to the uterus by the time of diagnosis, which corresponds to stage I according to FIGO classification. ${ }^{1}$ This was not the case of this patient, since peritoneal carcinomatosis and diffuse nodular hepatic metastases were documented. Therefore, search for other primary locations was performed using endoscopic procedure and imaging techniques. A 2003 review of 129 cases of uterine papillary serous carcinoma demonstrated that abnormal bleeding represents the presenting symptom in about two thirds of cases, being the most common also in other endometrial cancer subtypes; instead, abnormal Papanicolaou smear occurs isolated in 10\% and abdominal symptoms such as distension and/or pain in about $9 \%{ }^{3}$ In this patient, the presenting symptom was abdominal distension caused by moderate-volume ascites, along with anorexia and dyspnea.

Chylous ascites or chylaskos is a rare entity occurring in about 1 of 20,000 to 187,000 hospital admissions and being more common in females. It is defined as the accumulation of millky-appearing fluid containing high levels of triglycerides in the abdominal cavity. ${ }^{4}$ Staats has pointed a triglyceride level greater than $110 \mathrm{mg} / \mathrm{dL}$ to be highly sug- gestive of chylous ascites, even though arbitrary values have been set by different authors. ${ }^{5}$ On the other hand, Cárdenas et al have stated that ascitic fluid in chylaskos has the following characteristics: triglyceride level above $200 \mathrm{mg} / \mathrm{dL}$, cell count above 500 with predominance of lymphocytes, total protein between 2.5 and $7.0 \mathrm{~g} / \mathrm{dL}$, SAAG below $1.1 \mathrm{~g} /$ $\mathrm{dL}$ (except when cirrhosis is the cause of chylous ascites), LDH level between 110 and $200 \mathrm{IU} / \mathrm{L}$ and glucose level under $100 \mathrm{mg} / \mathrm{dL} .{ }^{7}$ In this case, all these criteria were fulfilled. Several causes have been described for chylous ascites. In developed countries, the main causes are malignancy or chronic hepatic diseases. On the other hand, infectious etiology and specifically tuberculosis is still very frequent. But other causes have been described, including inflammatory diseases, trauma, surgical iatrogenesis and congenital abnormalities, which must be considered particularly in infants. ${ }^{6}$ This patient had no history of liver disease, trauma or abdominal surgery and tuberculosis was also excluded by direct examination and culture of peritoneal fluid. In a 2011 systematic review on symptoms and causes of atraumatic chylous ascites, Steinemann et al reported that, in adults, malignancy is the cause in $17 \%$ of cases, with particular relevance for non-Hodgkin lymphoma. Endometrial cancer was reported only once as the cause of chylous ascites in 190 patients. ${ }^{4}$ The rarity of this presentation justifies the search for other potential etiologies of chylous ascites in this patient, but only one primary malignancy was able to explain the clinical scenario. To the best of our knowledge, only one case of chylous ascites caused by endometrial carcinoma has been published. ${ }^{8}$ Nevertheless, we do not know whether this case addresses a serous papillary adenocarcinoma or other type of endometrial carcinoma.

\section{References}

1. Ryan AJ, Susil B, Jobling TW, Oehler MK. Endometrial cancer. Cell Tissue Res 2005; 322:53-61.

2. Colombo N, Preti E, Landoni F, Carinelli S, Colombo A, Marini C et al. Endometrial cancer: ESMO Clinical Practice Guidelines for diagnosis, treatment and follow-up. Ann Oncol. 2013 Oct;24 Suppl 6:vi33-8

3. Slomovitz BM, Burke TW, Eifel PJ, Ramondetta LM, Silva EG, Jhingran A et al. Uterine papillary serous carcinoma (UPSC): a single institution review of 129 cases. Gynecol Oncol 2003;91:463-469.

4. Steinemann DC, Dindo D, Clavien PA, Nocito A. Atraumatic Chylous Ascites: Systematic Review on Symptoms and Causes. J Am Coll Surg 2011;212:899-905

5. Staats BA, Ellefson RD, Budahn LL, Dines DE, Prakash UB, Offord K. The lipoprotein profile of chylous and nonchylous pleural effusions. Mayo Clin Proc. 1980;55(11):700-4

6. Aalami 00 , Allen DB, Organ $\mathrm{CH}$ Jr. Chylous ascites: A collective review. Surgery 2000; 128: 761-78

7. Cárdenas A, Chopra S. Chylous ascites. Am J Gastroenterol 2002; 97:1896-1900

8. Garcia-RomanJM,DeAndresVidalS,DeLaFuenteBucetaA,Granados MartinMD.Endometrial carcinoma presenting with massive chylous ascites. Presse Med 1996;25:865 